SECTION 2. Applied mathematics. Mathematical modeling.

Shevtsov Alexandr Nikolayevich candidate of technical Sciences, President, Theoretical \& Applied Science, LLP, associate Professor of the Department «Applied mathematics» Taraz State University named after M.Kh. Dulati, Kazakhstan

Smailova Ylmeken Muhitovna candidate of physical and mathematical Sciences, associate Professor, Branch of JSC National centre of improvement of qualification of «Orleu» IPKPR regions of Zhambyl, Kazakhstan

Shyrynkhanova Dinara Zhaksylykovna 1 year magistr of the speciality "Information systems " Taraz State University named after M.Kh. Dulati, Kazakhstan

\title{
TIME CHARACTERISTICS OF SCRIPTS WORD IN DELPHI ENVIRONMENT
}

This article studies and develops methods of optimization of work processes and the choice of algorithms for processing of server scripts Word.

Keywords: optimization, Word, time.

\section{ВРЕМЕННЫЕ ХАРАКТЕРИСТИКИ ВЫПОЛНЕНИЯ СКРИПТОВ WОRD В СРЕДЕ ДЕЛЬФИ}

В данной статье рассматриваются методы оптимизачии процессов работы и выбора алгоритмов обработки скриптов сервера Word.

Ключевые слова: оптимизация, время.

Разработка программ зачастую связана с процессом оптимизации. Известно, что на этот этап разработки отводится больше всего времени. Рассмотрим программу в Delphi, основной функцией которой является обработка коллекций, их сравнение и формирование новой структуры данных в соответствии с поставленной блок схемой.

Тестирование будем вести на компьютере со следующими характеристиками (рис.1).

Для оценки времени выполнения отдельных скриптов используем алгоритм, привязанный к текущему времени системы.

Var

d: TDateTime;

$\mathrm{d}:=$ Now;

----------Скрипт-------------

Label14.Caption:=FormatDateTime('hh:mm:ss:zzz'， Now()-d); 


\begin{tabular}{|c|c|c|c|}
\hline Компонент & Подробно & Оценка & Общая оценка \\
\hline Процессор & Intel(R) Core(TM)2 Duo CPU T6600@2.20GHz & 5,7 & \\
\hline Память (03у) & 4,00 ГБ & 5,7 & \\
\hline Графика & $\begin{array}{l}\text { NVIDIA GeForce GT 240M (Microsoft } \\
\text { Corporation - WDDM v1.2) }\end{array}$ & 6,4 & \\
\hline Графика для игр & 2798 МБ графической памяти доступно & 6,4 & $\begin{array}{l}\text { Определяется } \\
\text { наименьшей } \\
\text { оценкой }\end{array}$ \\
\hline $\begin{array}{l}\text { Основной жесткий } \\
\text { диск }\end{array}$ & Свободно: 38GB (всего: 146GB) & 5,8 & \\
\hline
\end{tabular}

Система

$\begin{array}{ll}\text { Производитель } & \text { Acer } \\ \text { Модель } & \text { Aspire } 5739 \mathrm{G} \\ \text { Суммарный объем системной памяти } & 4,00 \text { ГБ 03У } \\ \text { Тип системы } & \text { 64-разрядная операционная система } \\ \text { Число ядер процессора } & 2\end{array}$

Рисунок 1 - Параметры компьютера.

Рассмотрим начальные скриптыи алгоритмы для соединения с сервером и настройки параметров ускорения обработки данных сервером Word 2007, и оценим их работу:

Таблица 1

\begin{tabular}{|l|l|l|}
\hline \multicolumn{1}{|c|}{ Скрипты } & \multicolumn{1}{|c|}{\begin{tabular}{c}
\multicolumn{1}{|c|}{ Время } \\
(сек.)
\end{tabular}} \\
\hline 1. & WordApp:=CreateOleObject('Word.Application'); & 0,521 \\
\hline 2. & WordApp.Documents.Open(FileName); & 0,677 \\
\hline 3. & WordApp.Visible:=false; & 0,051 \\
\hline 4. & WordApp.Options.CheckSpellingAsYouType:=False; & 0,015 \\
\hline 5. & WordApp.Options.CheckGrammarAsYouType:=False; & 0,002 \\
\hline 6. & WordApp.Options.CheckGrammarWithSpelling:= False; & 0,001 \\
\hline 7. & col:=WordApp.ActiveDocument.Paragraphs.Count; & 0,055 \\
\hline 8. & WordApp.Options.ContextualSpeller := False; & 0,016 \\
\hline 9. & WordApp.Options.ShowReadabilityStatistics := False; & 0,002 \\
\hline 10. & WordApp.ActiveDocument.ShowGrammaticalErrors := False; & 0,006 \\
\hline 11. & WordApp.ActiveDocument.ShowSpellingErrors := False; & 0,007 \\
\hline
\end{tabular}




\begin{tabular}{|l|l|l|}
\hline 12. & VarIsEmpty(WordApp); & 0,000 \\
\hline 13. & WordApp.Visible:=true; & 0,019 \\
\hline 14. & $\begin{array}{l}\text { WordApp.ActiveDocument.Close(SaveChanges,EmptyParam,EmptyPara } \\
\text { m); }\end{array}$ & 0,431 \\
\hline 15. & WordApp.Quit(SaveChanges,EmptyParam,EmptyParam); & 0,007 \\
\hline
\end{tabular}

Теперь рассмотрим скрипты непосредственной обработки коллекций и данных содержащихся в них, а также возможные способы их оптимизации.

Таблица 2

\begin{tabular}{|c|c|c|}
\hline & Скрипты & $\begin{array}{c}\text { Время } \\
\text { (сек.) }\end{array}$ \\
\hline 1. & OleContainer.copy; & 0,003 \\
\hline 2. & OleContainer1.paste; & 0,101 \\
\hline 3. & $\begin{array}{l}\text { OleContainer.copy; } \\
\text { OleContainer1.paste; } \\
\text { OleContainer2.paste; } \\
\text { OleContainer3.paste; } \\
\text { OleContainer4.paste; } \\
\text { OleContainer5.paste; }\end{array}$ & 0,455 \\
\hline 4. & application.ProcessMessages; & 0,005 \\
\hline 5. & v:=WordApp.ActiveDocument.Paragraphs.Item(1).Range.start; & 0,025 \\
\hline 6. & WordApp.ActiveDocument.Range(v,v+1).Select; & 0,015 \\
\hline 7. & s0:=WordApp.selection.text; & 0,011 \\
\hline 8. & $\begin{array}{l}\text { v:=WordApp.ActiveDocument.Paragraphs.Item(1).Range.start; } \\
\text { WordApp.ActiveDocument.Range(v,v+1).Select; } \\
\text { s0:=WordApp.selection.text; }\end{array}$ & 0,051 \\
\hline 9. & WordApp.selection.delete; & 0,002 \\
\hline 10. & WordApp.ActiveDocument.Range(v,v+1).Select; & 0,018 \\
\hline 11. & s0:=WordApp.selection.text; & 0,002 \\
\hline 12. & $\begin{array}{l}\text { while numb(s0) do } \\
\text { begin } \\
\text { WordApp.selection.delete; } \\
\text { WordApp.ActiveDocument.Range(v,v+1).Select; } \\
\text { s0:=WordApp.selection.text; } \\
\text { end; }\end{array}$ & 0,019 \\
\hline 13. & $\begin{array}{l}\text { for } \mathrm{k}:=1 \text { to } 3 \text { do begin } \\
\mathrm{v}:=\text { WordApp.ActiveDocument.Paragraphs.Item(2).Range.start; } \\
\text { WordApp.ActiveDocument.Range( } \mathrm{v}, \mathrm{v}+2) \text {.Select; } \\
\mathrm{s} 0:=\text { WordApp.selection.text; } \\
\text { if num(s0)=0 then } \\
\text { begin }\end{array}$ & 0,089 \\
\hline
\end{tabular}




\begin{tabular}{|l|l|l|}
\hline & $\begin{array}{l}\text { vk:=WordApp.ActiveDocument.Paragraphs.Item(1).Range.end; } \\
\text { WordApp.ActiveDocument.Range(vk-1,vk).Select; } \\
\text { WordApp.selection.delete; } \\
\text { end; } \\
\text { end; }\end{array}$ & \\
\hline 14. & WordApp.ActiveDocument.Paragraphs.Item(1).Range.select; & \\
\hline 15. & WordApp1.Selection.EndKey(wdStory,EmptyParam); & 0,021 \\
\hline 16. & WordApp1.Selection.InsertAfter(inttostr(n)+'. '); & 0,015 \\
\hline 17. & $\begin{array}{l}\text { WordApp1.Selection.EndKey(wdStory,EmptyParam); } \\
\text { WordApp1.selection.paste; }\end{array}$ & 0,002 \\
\hline 18. & $\begin{array}{l}\text { WordApp.Selection.EndKey(wdStory,EmptyParam); } \\
\text { WordApp.selection.paste; }\end{array}$ & 0,866 \\
\hline 19. & $\begin{array}{l}\text { WordApp1.Selection.EndKey(wdStory,EmptyParam); } \\
\text { case z of } \\
\text { 1:WordApp1.Selection.InsertAfter('A) '); }\end{array}$ & 0,0610 \\
\hline $\begin{array}{l}\text { 2:WordApp1.Selection.InsertAfter('B) '); } \\
\text { 3:WordApp1.Selection.InsertAfter('C) '); } \\
\text { 5:WordApp1.Selection.InsertAfter('D) '); } \\
\text { end; }\end{array}$ & \\
\hline
\end{tabular}

Интересные данные получим при выполнении скриптов внутри циклов, (табл. 2, пункт 19). Рассмотрим 5 вопросов, и 5 ответов, тогда получим временную матрицу:

Таблица 3

\begin{tabular}{|c|c|c|c|c|c|c|}
\hline & \multicolumn{6}{|c|}{ Вопросы } \\
\hline \multirow{6}{*}{$\begin{array}{l}\overrightarrow{\vec{E}} \\
\text { D. } \\
\tilde{0}\end{array}$} & & 1 & 2 & 3 & 4 & 5 \\
\hline & 1 & 0,010 & 0,014 & 0,027 & 0,023 & 0,031 \\
\hline & 2 & 0,022 & 0,021 & 0,032 & 0,032 & 0,034 \\
\hline & 3 & 0,033 & 0,022 & 0,032 & 0,034 & 0,024 \\
\hline & 4 & 0,020 & 0,023 & 0,022 & 0,028 & 0,024 \\
\hline & 5 & 0,029 & 0,031 & 0,021 & 0,041 & 0,032 \\
\hline
\end{tabular}

Аналогичным образом проходили аппробацию ряд тестов различного объема, и с различным контентом. В нескольких случаях наблюдался сбой работы буфера обмена, а также прерывание программы в месте нахождения разрыва строки, в конце одного из ответов, хотя первым из алгоритмов анализа как раз и является отыскание разрывов (Shift+Enter) и преобразование их в обычный переход на следующую строку (\#13\#10).

Делалось предположение о принадлежности распределения времени для скриптов находящихся в цикле к одному из характерных и известных ( нормальное, Пуассона, Лапласа и т.д.) но это не подтвердилось. 


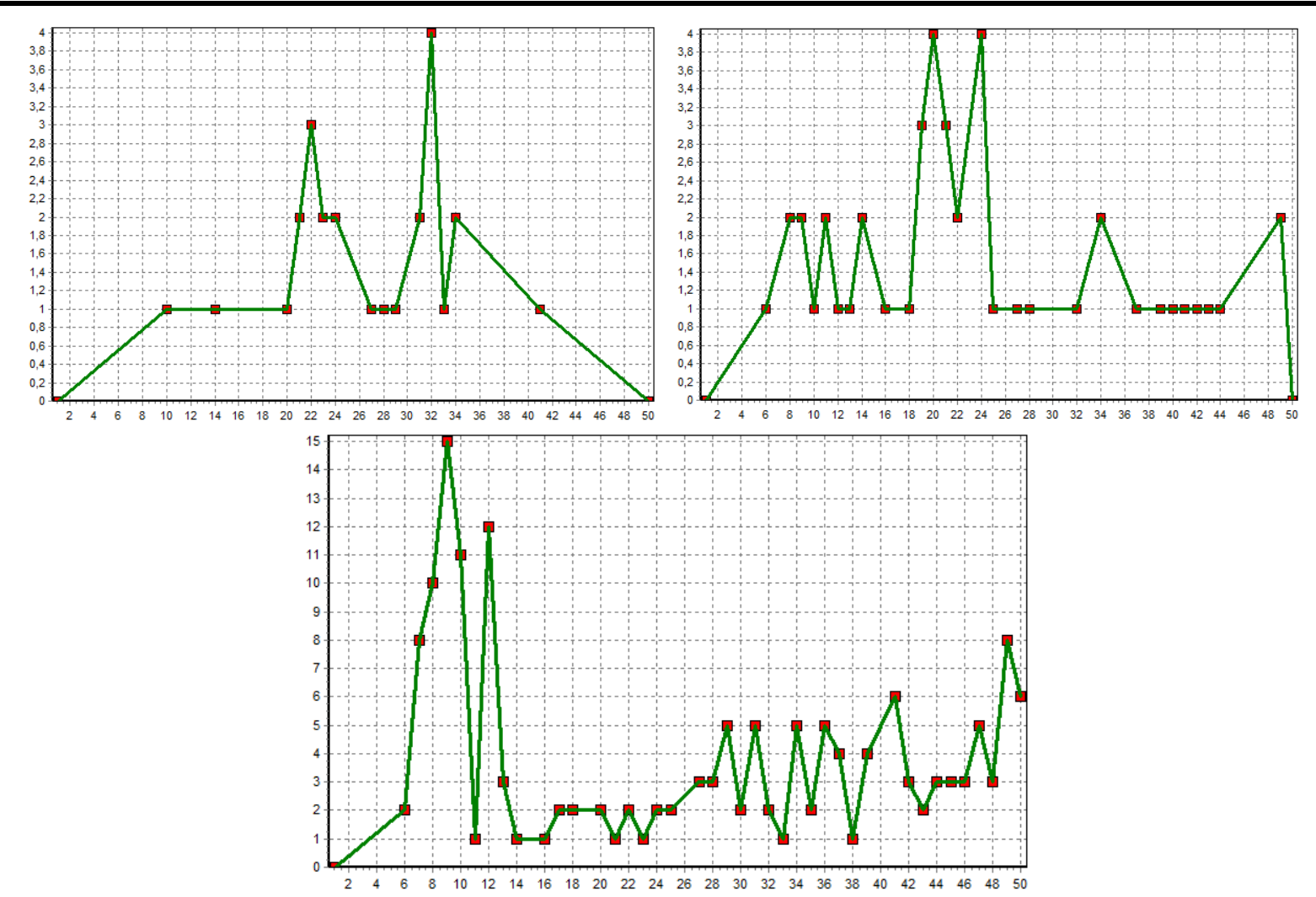

Рисунок 1 - График функции распределения. (5 вопросов, 10 вопросов, 70 вопросов)

Выводы:

- Прослеживается уменьшение времени при выполнении повторяющихся операций, (табл.2, пункты 1-3).

- В отдельных случаях одинаковые скрипты выполняются с разным временем, скорее всего, это связанно с объемом информации передаваемой через буфер обмена (табл.2, пункты 7 и 11).

- Время выполнения набора скриптов (табл.2, пункт 8) совпадает с сумарным временем выполнения отдельных скриптов (табл.2, пункты 5-7).

- Проверка условия (табл.2, пункт 12), и отказ от выполнения алгоритма, может занимать столько же системного времени как и сам вложенный исполняемый алгоритм.

- Расчеты в пределах одного сервера выполняются быстрее (табл.2, пункт 13), особенно это заметно при копировании информации через буфер обмена и вставке в параллельный сервер (табл.2, пункты 17 и 18), разница во времени составляет 0,805 секунды, что весьма значительно, и должно быть учтено при оптимизации.

- Построенная функция распределения не дает четкой зависимости времени от каких либо параметров, также не выявлена зависимость от размера объектов помещаемых в буфер.

\section{Литература:}

1. Шевцов А.Н., Шырынханова Д.Ж. Разработка алгоритмов и приложения компонентной модели для анализа и исправления ошибок экзаменационного теста. Theoretical \& Applied Science. «Development of Applied Mathematics», ISPC, 30.05.2013, Taraz, Kazakhstan. - №5, 2013. -p.77-83. 
2. Шевцов А.Н., Смайлова У.М., Шырынханова Д.Ж. Некоторые алгоритмы предварительной обработки теста. Theoretical \& Applied Science. «Results \& Perspectives», ISPC, 30.09.2013, Florence, Italy. - №9, 2013. -p.51-58.

\section{Текст программы}

\section{Приложение 1}

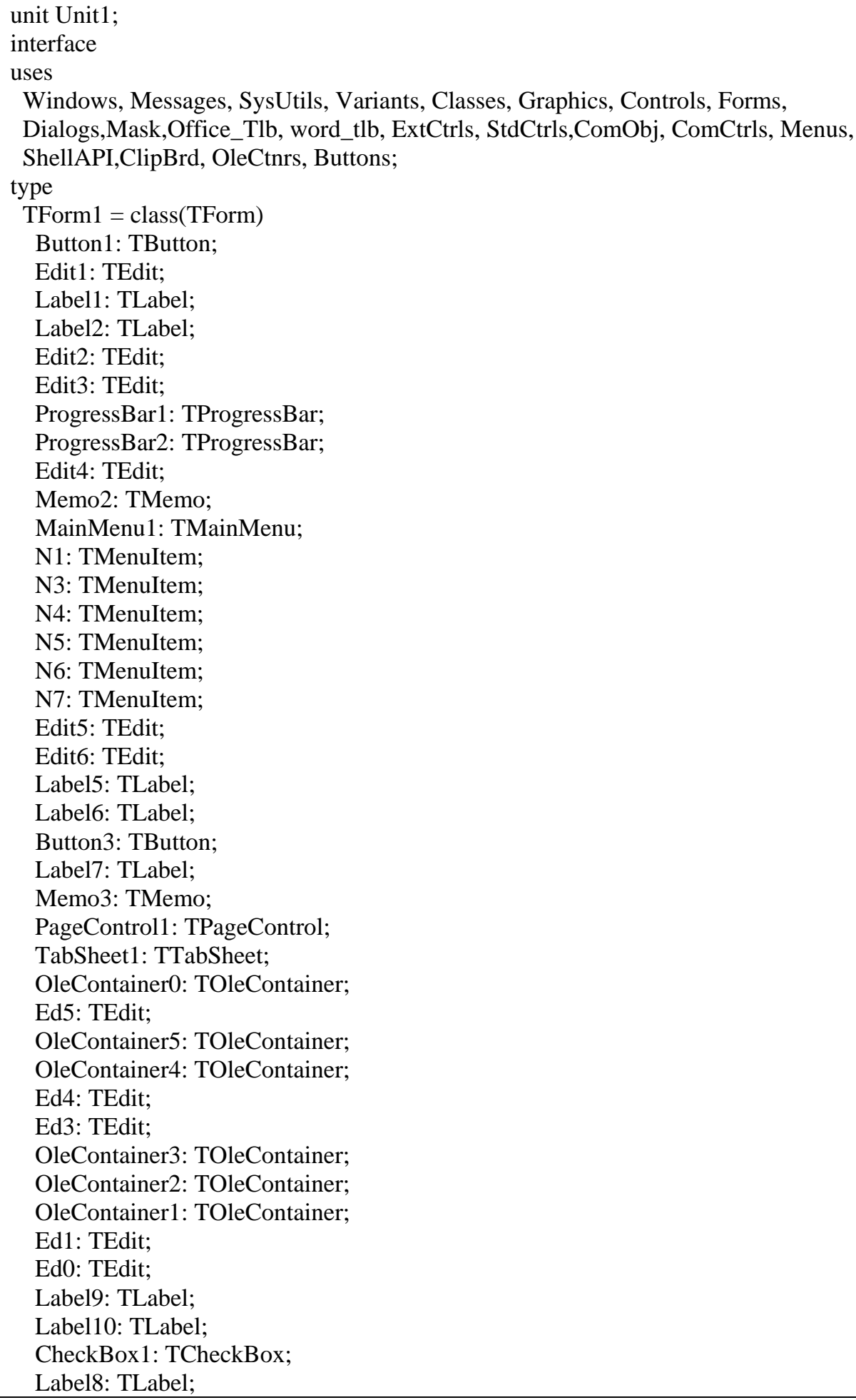




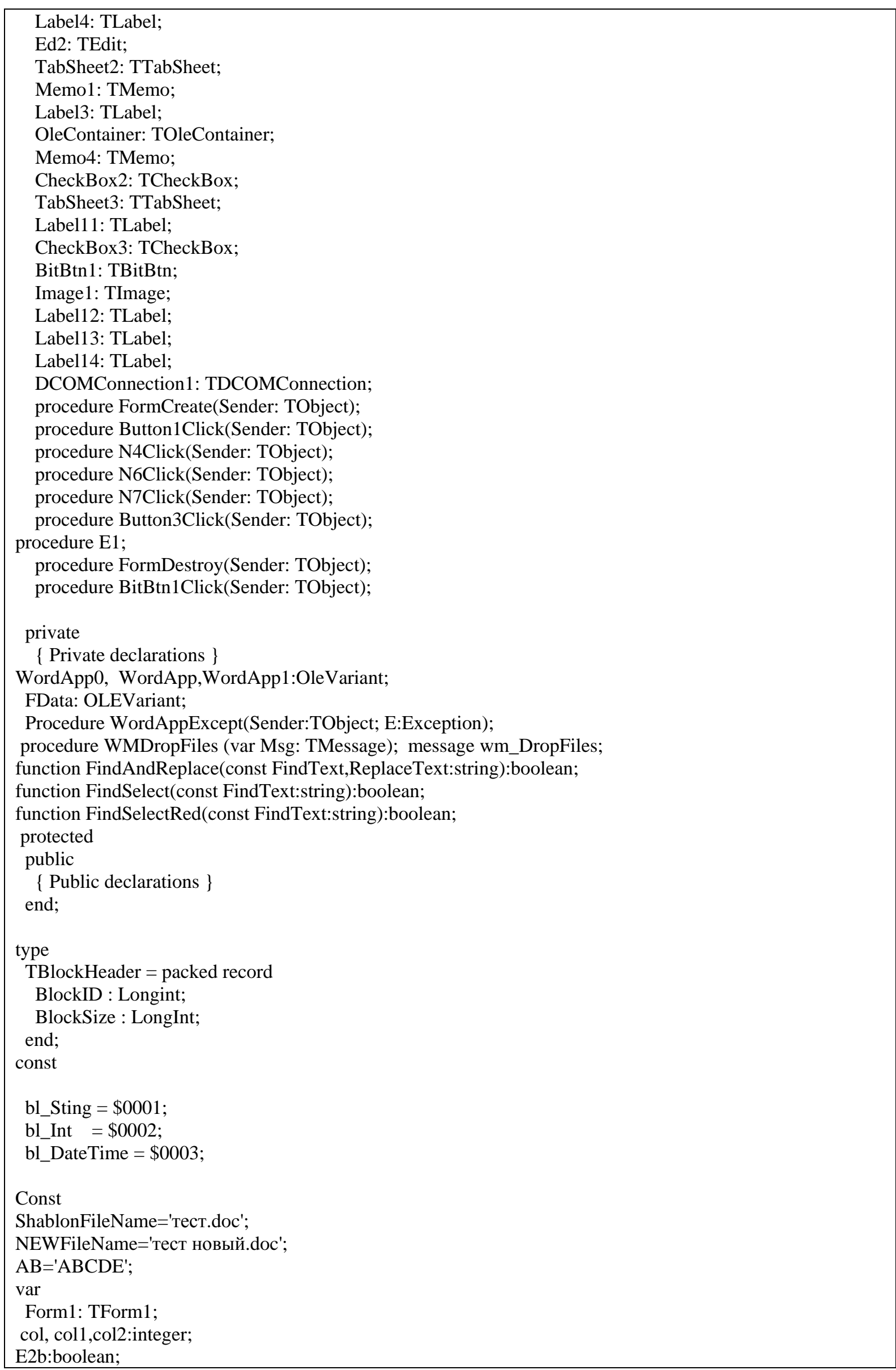




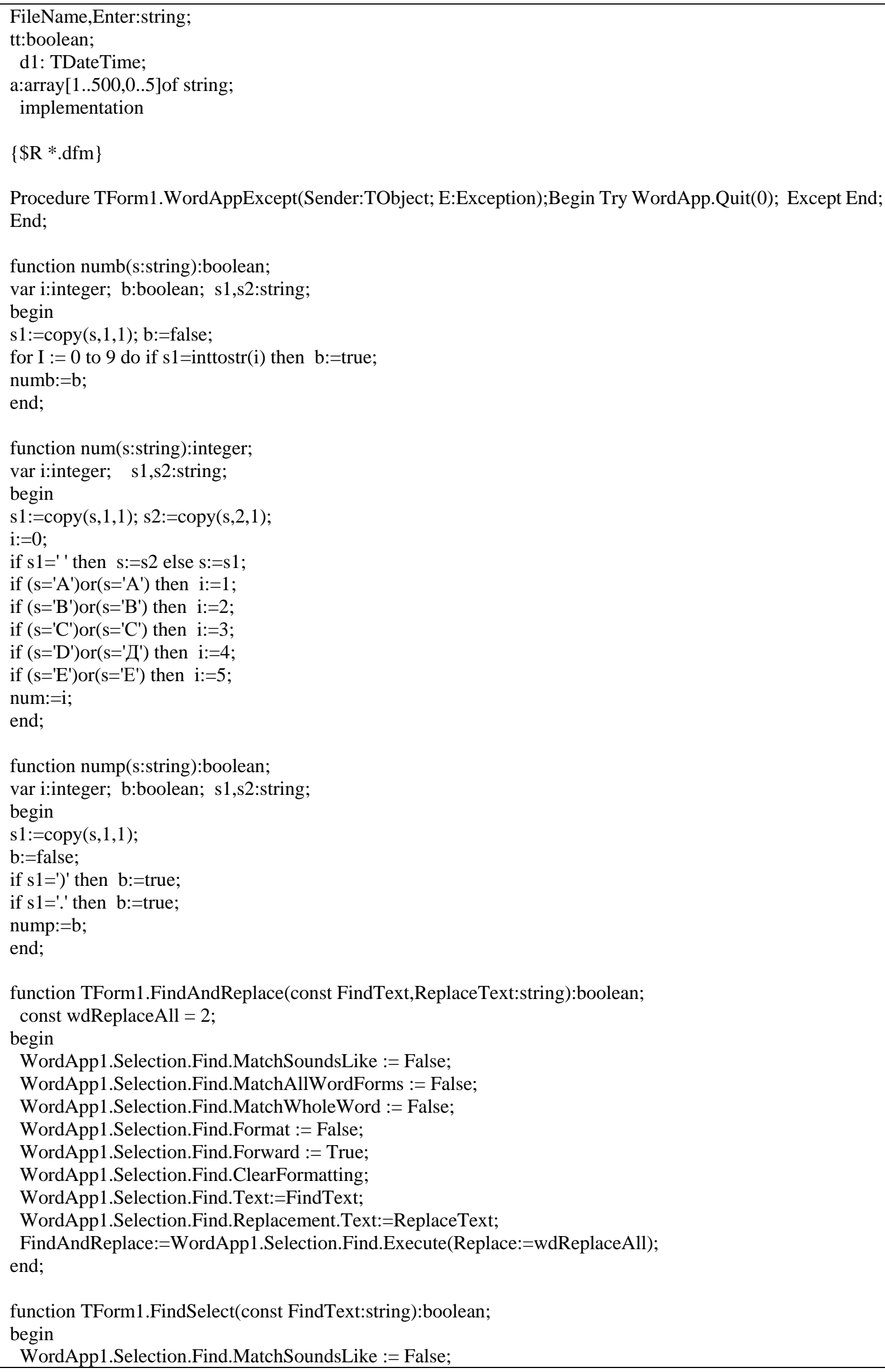




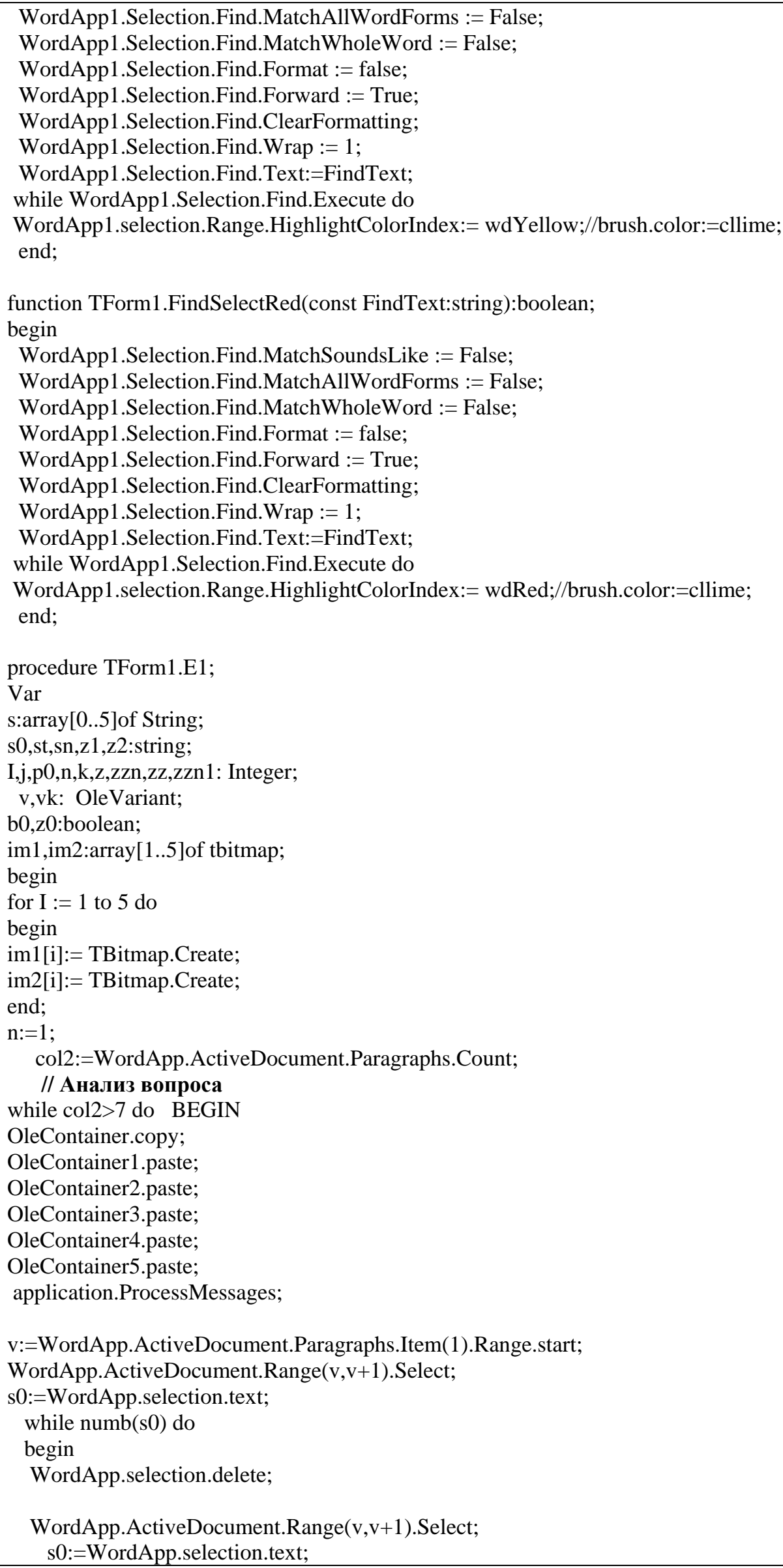




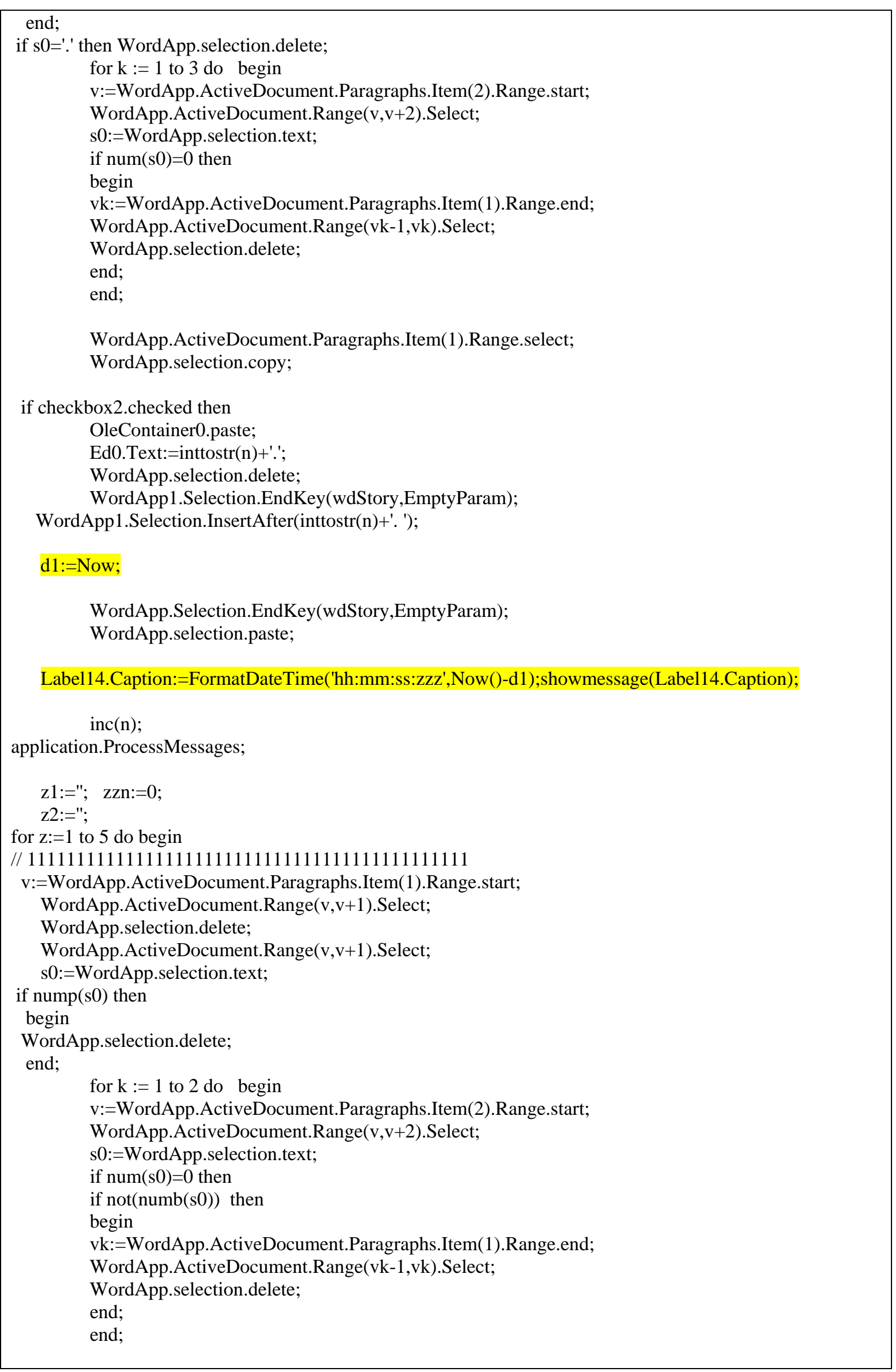


v:=WordApp.ActiveDocument.Paragraphs.Item(1).Range.start; vk:=WordApp.ActiveDocument.Paragraphs.Item(1).Range.end; WordApp.ActiveDocument.Range(v,vk).Select;

WordApp.selection.copy;

if checkbox2.checked then

case $\mathrm{z}$ of

1:OleContainer1.paste;

2:OleContainer2.paste;

3:OleContainer3.paste;

4:OleContainer4.paste;

5:OleContainer5.paste;

end;

WordApp1.Selection.EndKey(wdStory,EmptyParam);

case $\mathrm{z}$ of

1:WordApp1.Selection.InsertAfter('A) ');

2:WordApp1.Selection.InsertAfter('B) ');

3:WordApp1.Selection.InsertAfter('C) ');

4:WordApp1.Selection.InsertAfter('D) ');

5:WordApp1.Selection.InsertAfter('E) ');

end;

WordApp1.Selection.EndKey(wdStory,EmptyParam);

if numb(s0)and $(\mathrm{z}<5)$ then

begin

WordApp1.Selection.InsertAfter(' //Нет ответа//'+\#13\#10);

WordApp.Selection.HomeKey(wdStory,EmptyParam);

WordApp.Selection.Insertbefore('A) '+\#13\#10);

v:=WordApp.ActiveDocument.Paragraphs.Item(2).Range.start;

WordApp.ActiveDocument.Range(v,v).Select;

WordApp.Selection.Insertbefore('A) ');

v:=WordApp.ActiveDocument.Paragraphs.Item(1).Range.start; vk:=WordApp.ActiveDocument.Paragraphs.Item(1).Range.end; WordApp.ActiveDocument.Range(v,vk).Select;

WordApp.selection.delete;

end

else

begin

$\mathrm{v}$ :=WordApp.ActiveDocument.Paragraphs.Item(1).Range.start;

vk:=WordApp.ActiveDocument.Paragraphs.Item(1).Range.end;

WordApp.ActiveDocument.Range(v,vk).Select;

WordApp1.selection.paste;

if $\mathrm{z}=1$ then

begin

z1:= WordApp.selection.text;

zzn:=WordApp.Selection.InlineShapes.count;

if $\mathrm{zzn}>0$ then

for $\mathrm{zz}:=1$ to zzn do

begin

WordApp.Selection.Range.InlineShapes.Item(zz).Select;//CopyAsPicture;

WordApp.Selection.Copy;

Im1[zz].Assign(Clipboard);

end;

end; 


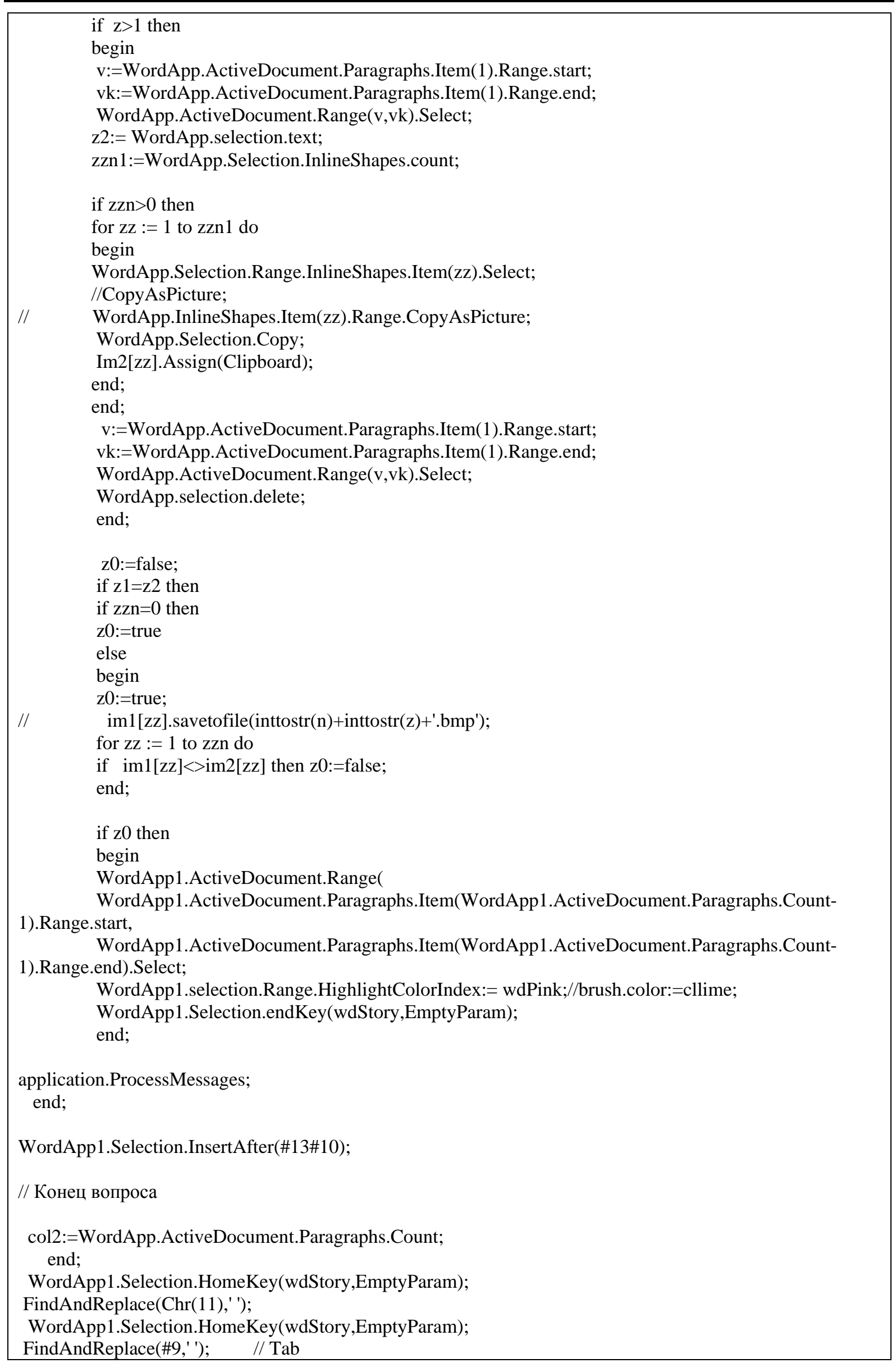




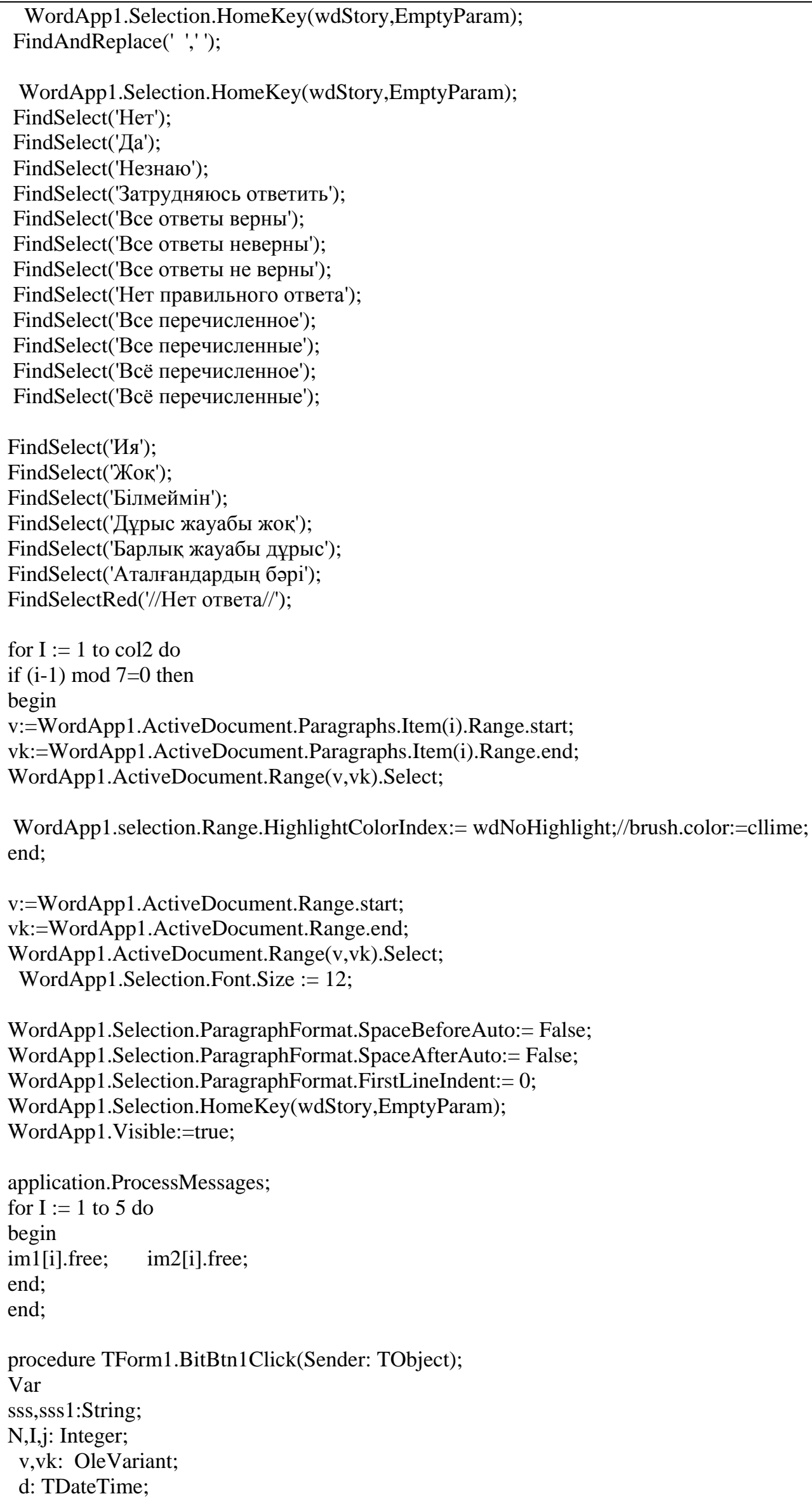




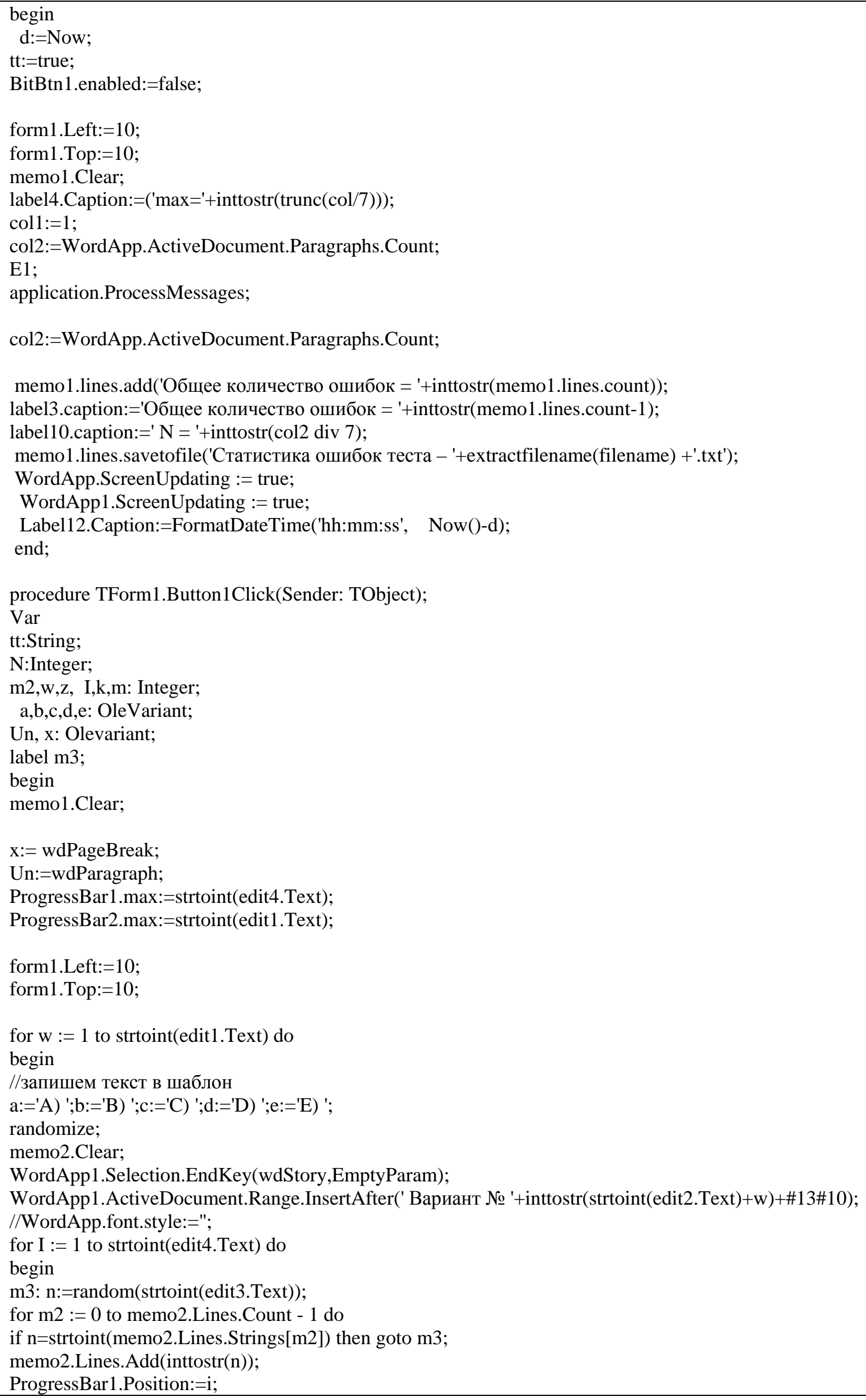




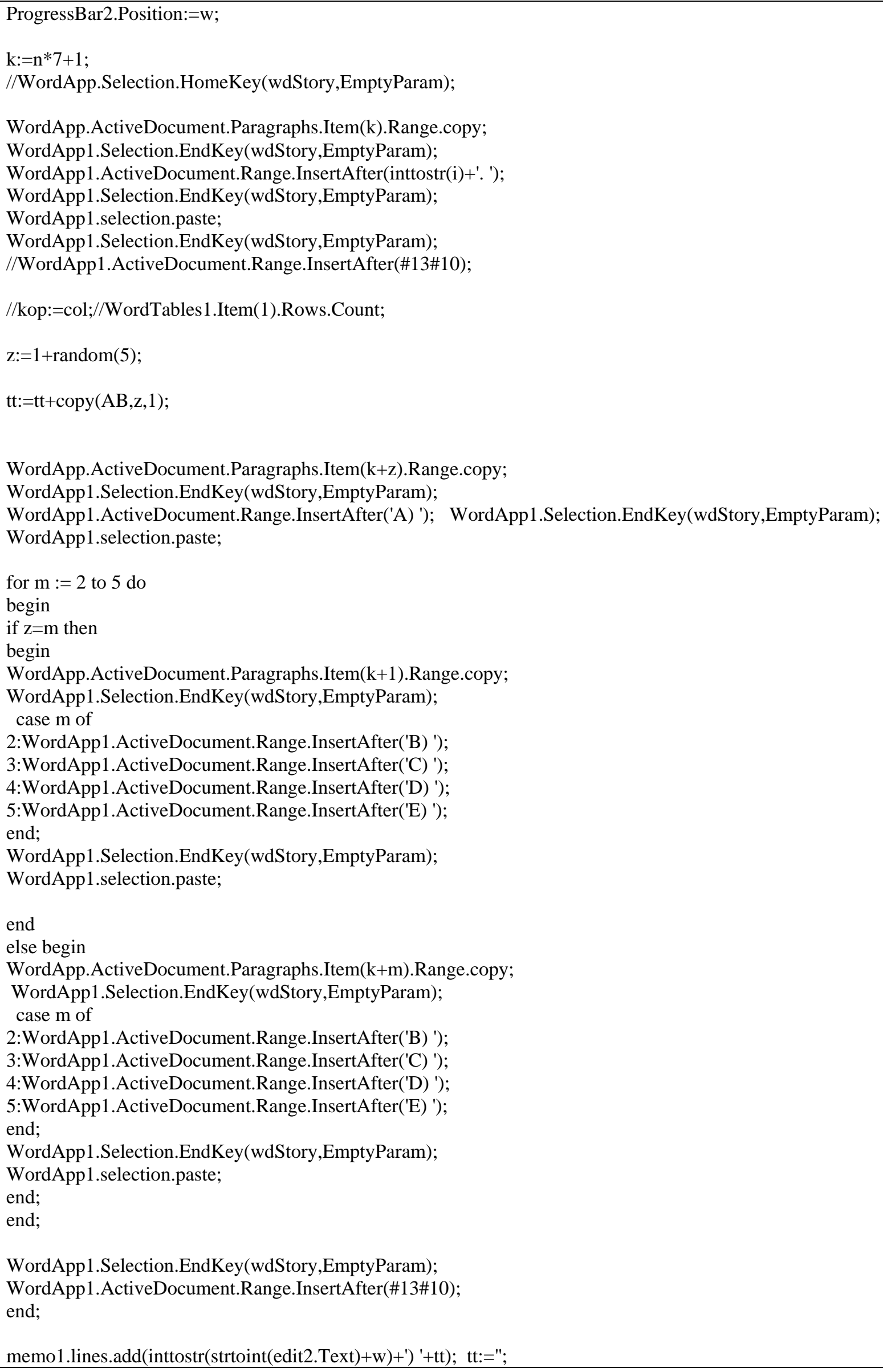




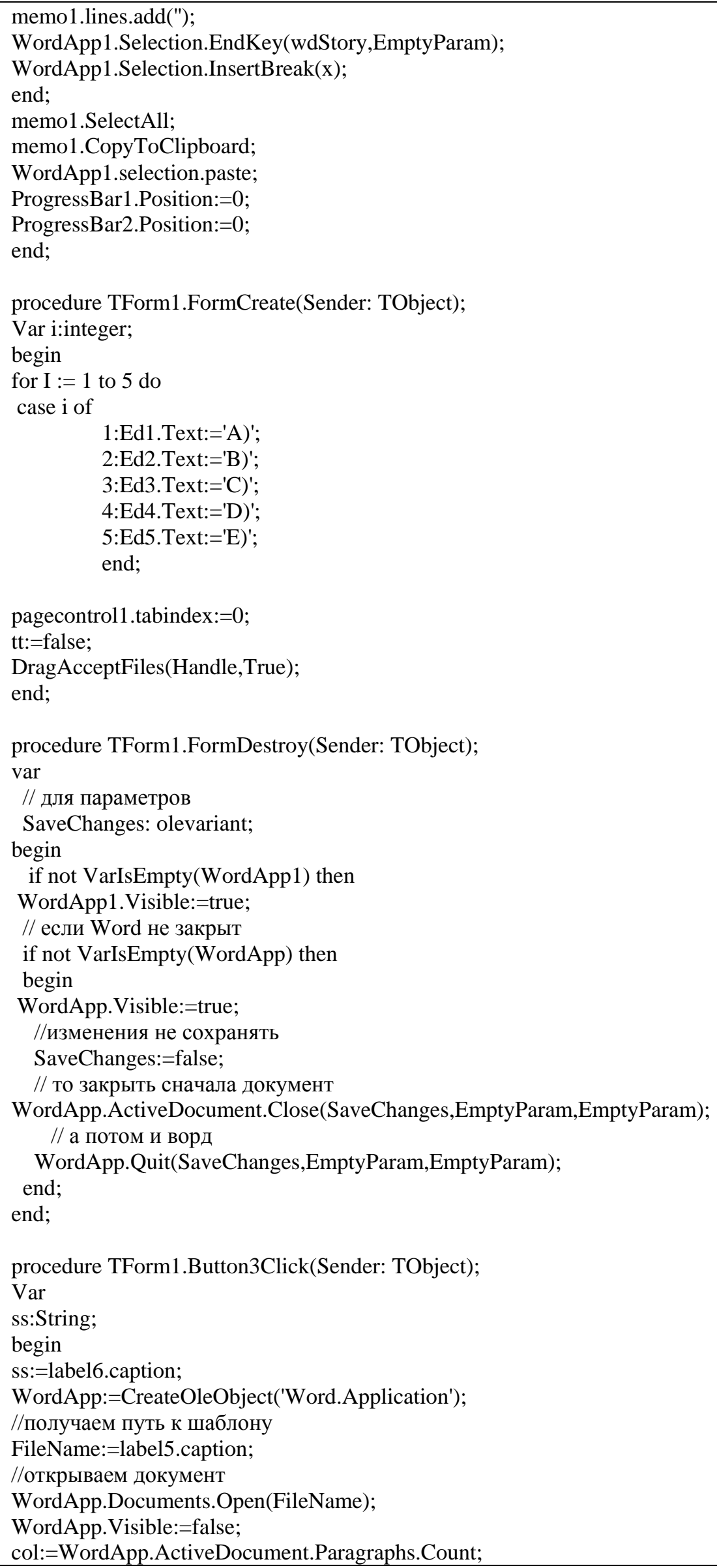




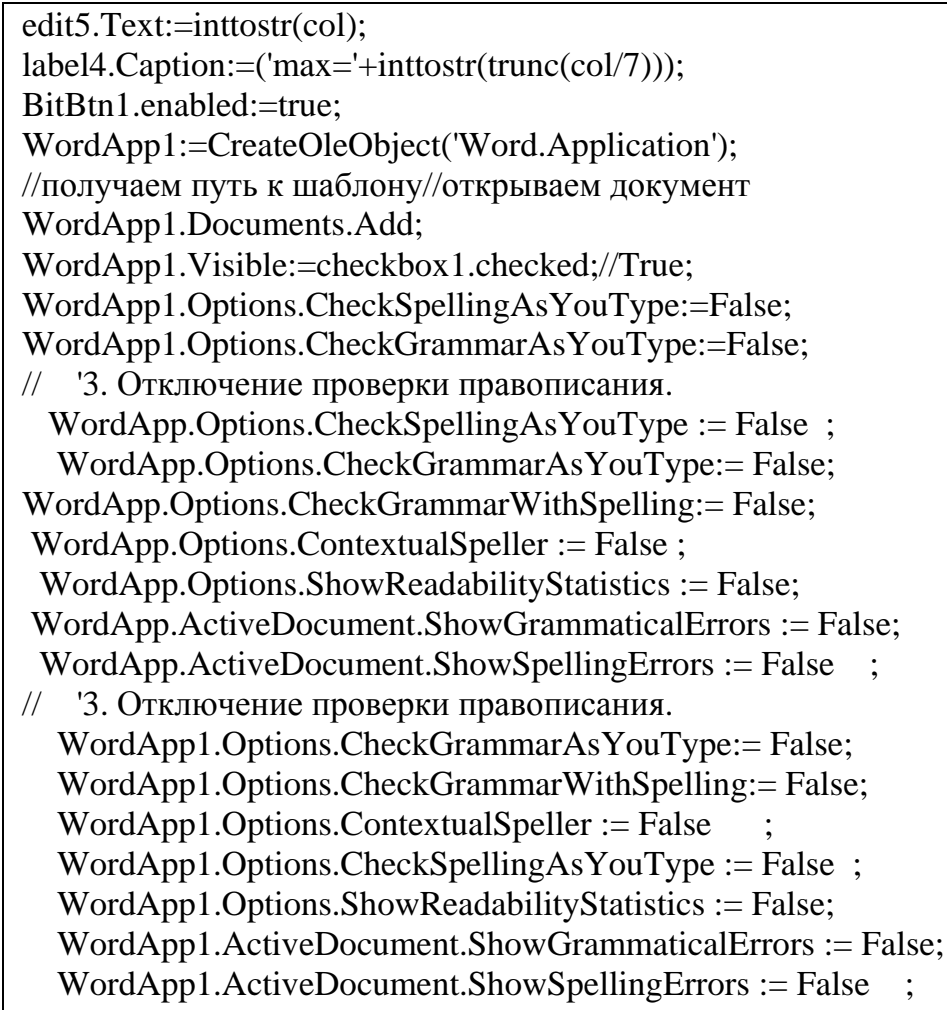

if checkbox3.checked then BitBtn1.click;

WordApp.Visible:=true;

WordApp1.Visible:=true;

end;

procedure TForm1.WMDropFiles(var Msg: TMessage);

Var Filename: array[0..256] of char;

begin

DragQueryFile(THandle(Msg.WParam),0,Filename,SizeOf(Filename));

// Теперь в переменной Filename будет находиться путь

// к перетаскиваемому файлу. Далее вы можете выполнять с этим файлом, зная

// его путь, все что угодно.

label5.caption:=FileName;

label6.caption:=extractfilename(label5.caption);

label11.caption:=copy(extractfilename(label5.caption),1,length(extractfilename(label5.caption))-4);

Button3.Click;//BitBtn1.Click;

//Сообщаем об окончании претаскивания

DragFinish(THandle(Msg.WParam)); end;

procedure TForm1.N4Click(Sender: TObject);

begin

application.Terminate;

end;

procedure TForm1.N6Click(Sender: TObject);

begin showmessage('Программа предназначенна для предварительного анализа и автоматического
исправления ошибок экзаменационных тестов...');

end;

end. 